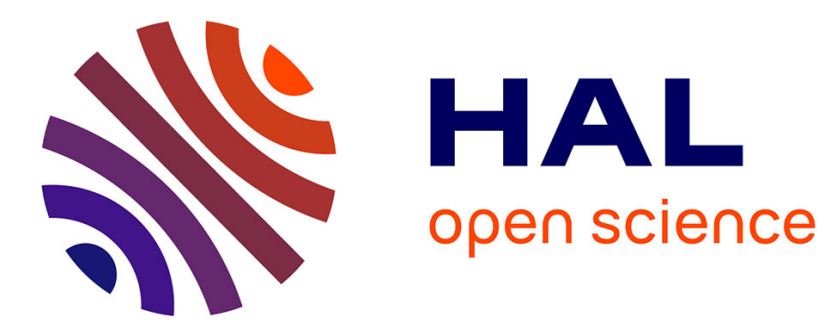

\title{
Emplois verbaux, distributions, métaphores
}

Christian Leclère

\section{To cite this version:}

Christian Leclère. Emplois verbaux, distributions, métaphores. Langue francaise, Paris : Larousse, 2002, 134 (134), pp.78-89. hal-00639302

\section{HAL Id: hal-00639302 https://hal.science/hal-00639302}

Submitted on 8 Nov 2011

HAL is a multi-disciplinary open access archive for the deposit and dissemination of scientific research documents, whether they are published or not. The documents may come from teaching and research institutions in France or abroad, or from public or private research centers.
L'archive ouverte pluridisciplinaire HAL, est destinée au dépôt et à la diffusion de documents scientifiques de niveau recherche, publiés ou non, émanant des établissements d'enseignement et de recherche français ou étrangers, des laboratoires publics ou privés. 


\title{
Emplois verbaux, distributions, métaphores
}

\author{
In: Langue française. $\mathrm{N}^{\circ} 134,2002$. pp. 78-89.
}

\section{Abstract \\ Christian Leclère : Verbal Constructions and methaphorical uses}

The Lexicon-Grammar, built at LADL, attemps to classify the syntactic structures of French and the set of relations which hold between them by means of the lexical elements which admit or exclude these syntactic structures. Thousands of French simple verbs have been classified, according to the syntactic structures of the sentences in which they may occur. They have been grouped within 60 syntactic tables.

Each verbal entry in a table includes the structure of a "defining sentence", characterizing the particular use of the word which the entry relates to and the particular distribution of arguments that are involved. Constructions associated with this defining construction (specific distributions, substructures, metaphors, etc.) are taken into account in the description, and noted as properties of each particular entry.

A verb has as many entries as it has uses that are judged to be distinct. In many cases, this distinction is difficult to establish. It depends on the answer to the question: does a variation in the distributional features of the argument create a different use, which deserves another entry, or is it a metaphor which can be noted as a property of the entry in question? This article tries to answer this question... and fails to do so.

Citer ce document / Cite this document :

Leclère Christian. Emplois verbaux, distributions, métaphores. In: Langue française. №134, 2002. pp. 78-89.

doi : $10.3406 /$ lfr.2002.6454

http://www.persee.fr/web/revues/home/prescript/article//fr_0023-8368_2002_num_134_1_6454 


\section{EMPLOIS VERBAUX, DISTRIBUTIONS, MÉTAPHORES ${ }^{1}$}

On peut facilement se méprendre quant à l'espèce de la métaphore

(P. Fontanier)

\section{Introduction}

Les différentes entrées d'un mot dans un dictionnaire correspondent à des sens jugés différents par le lexicographe. Elles se distinguent souvent par des variations de structure des phrases dans lesquelles le mot peut entrer et de distribution des actants dans ces phrases. Un sens métaphorique est le plus souvent décrit comme une acception déviante par rapport au sens "propre", «basique » ou "normal " du mot considéré.

Les classifications établies au LADL, appuyées sur des critères syntaxiques et distributionnels, ont abouti à la constitution d'un Lexique-Grammaire, dans lequel chaque entrée correspond à un ensemble de constructions possibles d'un mot dans un sens donné. Il décrit en outre les relations qu'entretiennent entre elles ces constructions. Quand un mot fait l'objet de plusieurs entrées, les dédoublements doivent se justifier par des critères formels aussi précis que possible. Je tenterai ici de poser certaines questions relatives aux relations métaphoriques à l'intérieur de ce cadre théorique, en me limitant aux entrées verbales simples.

\section{Critères de dédoublement}

Définir un emploi verbal, c'est :

a) déterminer l'ensemble des constructions qui caractérisent le fonction-

1. Je remercie Nicolette Le Dévéhat pour son soutien constant depuis plus de 30 ans. Mes recherches doivent beaucoup aux "séminaires du mardi", dans lesquels elle a eu un rôle discret mais déterminant. 
nement d'un verbe, c'est-à-dire la structure des phrases où il peut entrer, et la nature et la place de ses arguments essentiels dans ces phrases;

b) décrire les relations qu'entretiennent entre elles ces constructions (effacement, déplacement, permutation d'actants, etc.) et les variations de sens qu'elles entraînent (variations aspectuelles, ellipses, etc.).

Dans toutes les constructions qui définissent un tel emploi verbal, le verbe a un même "sens " nucléaire. Cette notion intuitive doit être "reproductible " chez une majorité de locuteurs.

Dans la classification du Lexique-Grammaire, une construction, appelée «définitionnelle», est considérée comme représentative de l'ensemble des constructions définissant chaque emploi verbal. En principe, c'est celle qui contient le maximum d'informations considérées comme cruciales pour délimiter le sens de l'emploi et décrire les variations syntaxiques et distributionnelles des différentes constructions concernées. La construction définitionnelle détermine l'entrée dans une table syntaxique, laquelle regroupe tous les verbes ayant cette construction (une soixantaine de tables pour quelque 15000 entrées de verbes simples).

\subsection{Variations de structure et dédoublement}

Un verbe a autant d'emplois, et donc autant d'entrées dans les tables, qu'on a trouvé de groupes de constructions définissant des sens différents. On n'a qu'une seule entrée pour un verbe qui a le même sens dans toutes ses constructions (c'est le cas de beaucoup de verbes techniques, comme capsuler, par exemple), et plusieurs entrées (jusqu'à des dizaines) pour les verbes très peu spécialisés (comme compter ou jouer).

Dans les cas les plus simples, deux sens différents d'un verbe correspondent à des structures formelles de phrases différentes, faciles à établir. Le verbe emprunter, par exemple, a deux emplois correspondant à deux structures (définitionnelles) nettement distinctes et aura deux entrées dans les tables (respectivement 36DT et $38 \mathrm{~L} 1$ ) :

(1) Paul emprunte mille francs à Jean

(2) Pierre a emprunté une route secondaire

NO V N1 Prép N2

NO VN1

Dans le cas suivant du verbe enrober, les deux structures sont du même type, mais elles sont reliées entre elles:
a. Luc a enrobé les bonbons de chocolat
b. Le chocolat enrobe les bonbons
No V N1 Prép N2
NO VN1

La phrase (3b) peut être considérée comme dérivée de (3a) : la relation est productive et connue. La construction (3a) est définitionnelle de cet emploi de enrober (table 37M6) et la possibilité d'avoir (3b) sera notée N2 V N1 dans cette table, pour marquer la possibilité de passage du complément $N 2$ en position 
sujet. Cette opération est courante pour les verbes de cette classe et les variations de sens en sont connues (absence du sujet actif et interprétation "statique » ou "résultative " de la phrase). Les deux constructions sont donc considérées comme relevant d'un même emploi et font l'objet d'une seule entrée.

\subsection{Variations de distribution et dédoublement}

La structure des phrases n'est pas un critère suffisant : les variations de distribution sont cruciales pour distinguer des emplois différents, et donc déterminantes pour la répartition en classes : d'où la présence de traits distributionnels dans les constructions définitionnelles des tables. Les deux phrases:

(1) Paul a chiffonné la feuille de papier

(2) La nouvelle a chiffonné Anne

correspondent nettement à deux sens du verbe chiffonner. Elles ont la même structure NO VN1, mais si l'on précise que l'objet direct dans (1) ne peut être qu'un nom «non humain concret " et celui de (2) qu'un nom «humain» (ces deux notions peuvent être confirmées par des tests formels comme la question en "qui ?»), on peut justifier un dédoublement des entrées qui rendra compte de l'intuition nette de différence de sens entre les deux emplois (tables 32CV et 4 respectivement).

Pour classifier l'ensemble des verbes, il a fallu faire une sélection de propriétés distributionnelles susceptibles de justifier des dédoublements d'entrées intéressant l'ensemble du corpus. Ces propriétés doivent répondre à des critères formels aussi nets que possible d'une part, et d'autre part aboutir à des sous-classes sémantiquement homogènes, pour former des «classes naturelles » de verbes assez larges, susceptibles d'études ultérieures plus fines $^{2}$. Des notions comme humain/non humain, concret/abstrait, pluriel obligatoire, etc. répondent aux exigences de la classification générale. Elles permettent de faire entrer dans des tables différentes, par exemple:
Max a violé une adolescente
$(N 1=$ Nhum pur, table $32 \mathrm{H})$
Max a violé la loi du silence
(N1 = Nabstrait, table 32R3)
Luc a gobé l'huître
$(N 1=$ Nconcret, table 38L0)
Luc a gobé (mon histoire + que Léa avait divorcé) $\quad(N 1=$ Nabstrait ou complétive, table 6)

Des critères distributionnels peuvent aussi servir à dédoubler l'entrée d'un verbe dans une même table: les deux emplois, correspondant à deux sens

2. On peut par exemple définir précisément l'objet direct du chiffonner de (4): on aboutit alors à une série de traits sémantiques définissant une "classe d'objet " du type de celles constituées par Gaston Gross (1994), mais ces traits n'intéresseraient ici qu'une minorité de verbes et ne peuvent pas être retenus dans la classification générale des emplois verbaux, sous peine d'une multiplication trop importante des propriétés, entraînant une multiplication des classes de verbes. 
différents, ont la même construction définitionnelle, mais une propriété distributionnelle "secondaire" (codée dans la table) permet de les distinguer. Par exemple dans la table 36DT, définie par NO VN1 à N2, on trouve deux entrées de donner:

Paul a donné un bouquet à Ida $\quad(N 1=$ Nconcret $)$

Paulo a donné ses complices aux flics $\quad(N 1=$ Nhumain $)$

\subsection{Dédoublements structurels et distributionnels}

C'est le cas le plus fréquent: lorsqu'un verbe a plusieurs emplois, il se comporte différemment, syntaxiquement et distributionnellement, selon le sens dans lequel il est pris. Le cas est clair pour les deux verbes projeter suivants, par exemple:

(1) Paul projette (un voyage + de partir) au Pérou

(2) Luc a projeté du vin sur le mur

La nature de l'objet direct est différente: $N$ prédicatif pour (1), $N$ concret pour (2) et les constructions sont différentes: complément locatif pour (2), qu'on ne trouve pas pour (1). De plus, les nominalisations des verbes apportent un argument supplémentaire pour justifier un dédoublement : projet pour (1), projection pour (2).

D'autres cas sont moins clairs: les deux constructions $N O V$ Loc N1 et $N O V$ N1 Loc N2, par exemple (où $L o c=$ préposition locative), correspondent respectivement à la définition des tables 35L et 38LD. Les verbes aboutir et pousser en sont des exemples:

(1) Le bateau a abouti sur la plage (table 35L)

(2) Paul a poussé le bateau sur la plage (table 38LD)

Dans cette dernière table (38LD), un codage prévoit la possibilité de passage de l'objet direct en position sujet (cette propriété, N1 V Loc N2, est partagée par plusieurs verbes de la table):

(3) a. Paul a échoué le batcau sur la plage (38LS)

b. Le bateau a échoué sur la plage

Bien que (3b) ait la même structure que (1), la phrase peut être considérée comme dérivée de (3a): elle ne fait donc pas l'objet d'une entrée dans la table 35L. Par contre, dans la phrase (4), échouer correspond bien à une entrée dans la table $35 \mathrm{~L}$; le sens différent du verbe s'accompagne d'un blocage de la transformation - on ne peut dériver (4) de (5):

(4) Paul a échoué dans un taudis; le coffre a échoué au grenier

(5) *On a échoué Paul dans un taudis; * on a échoué le coffre au grenier

Le verbe échouer sera donc dédoublé et figurera dans les deux tables 38LD et 35L. A priori, les phrases avec échoner de structure $N V$ Loc $N$ sont donc ambiguës : il peut s'agir soit de l'entrée du échouer $35 \mathrm{~L}$, soit d'une construction dérivée du échouer $38 \mathrm{LD}$. Mais à y regarder de plus près, la construction 
transitive ( $3 a)$, et par conséquent la relation ( $3 a-3 b)$, ne sont possibles que dans le cas où $N 1$ est un bateau. Encore n'est-il pas clair que (3b) corresponde au sens de (3a) : en langage marin, la forme pronominale Le bateau s'est échoué sur la plage est plus naturelle. Auquel cas, (3b) ne serait qu'un cas particulier de l'emploi de type (4) et il n'y aurait pas de relation entre les deux verbes échouer.

\section{La place de la métaphore}

Les dédoublements d'entrées sont fonction de critères syntaxiques et distributionnels, j'en ai donné plusieurs exemples. Une fois établis les différents emplois d'un verbe à partir de ces critères, on peut définir la métaphore comme une violation, pour un emploi donné, des critères choisis pour définir cet emploi. On voit bien la faille : la métaphore est définie comme une variation par rapport à une distribution "normale " et elle entraîne souvent des variations syntaxiques ( $c f$. Boons 1971, Gross 1975). Or les variations syntaxiques constituent des critères qui justifient les dédoublements d'entrées. Une variante métaphorique répond donc à tous les critères qui justifient un dédoublement. Mais effectuer ce dédoublement, c'est décider qu'on a un autre emploi, c'est-à-dire un autre verbe, et non une métaphore. Poussé à l'extrême, cette démarche aboutirait à créer une entrée autonome pour toute métaphore trouvée, c'est-à-dire à considérer qu'il n'y a pas de métaphores, mais seulement des emplois distincts.

\subsection{Dédoublement plutôt que propriété métaphorique}

Le problème du classement des emplois verbaux consiste, on l'a vu, à regrouper sous une même entrée les différentes réalisations syntaxiques concernant chaque sens d'un verbe, métaphorique ou non, et à décider de la frontière au-delà de laquelle on a affaire à un autre sens et à d'autres réalisations syntaxiques justifiant la création d'une autre entrée. L'un des emplois peut être clairement un emploi "figuré ", né d'une métaphore, mais il s'agit là d'une considération historique : synchroniquement, on considérera qu'il s'agit de deux emplois autonomes.

Prenons l'exemple du verbe désarmer dans les phrases suivantes :

(1) Les flics ont désarmé le forcené

(2) Les arguments de Paul ont désarmé Marie

L'intuition nette est que la phrase (1) correspond au sens "propre " du verbe ("enlever son/ses arme(s) à quelqu'un»). La construction est définitionnelle de la table 37E du Lexique-Grammaire. On pourrait défendre l'idée, sans doute exacte historiquement, que (2) constitue un emploi métaphorique de (1). Il y a violation d'une contrainte distributionnelle : l'emploi de base (1) 
exige un sujet «humain », alors que (2) accepte, outre un sujet humain, un sujet non humain ou même complétif (Que Marie ait dit cela a désarmé Paul).

Nous avons, dans ce cas, préféré dédoubler l'entrée, c'est-à-dire considérer qu'il y avait deux verbes désarmer.

Une première raison en est que l'intuition de différence de sens est assez forte. Il s'agit là évidemment d'un critère intuitif qui dépend de l'idée qu'on se fait de la notion de sens : sur quelle base appuyer cette intuition ? On pourrait envisager de définir un sens "nucléaire" du verbe, qui représenterait ce que tous les emplois, propres, figurés ou métaphoriques d'un même verbe ont en commun. Il n'y aurait alors qu'une seule entrée, et toutes les distributions possibles seraient représentées comme des propriétés de cette entrée. Ce sens "nucléaire " correspond le plus souvent à une hypothèse abstraite de type logique, difficile à formuler. Cette solution, envisageable dans l'optique d'une description purement sémantique, n'est évidemment pas satisfaisante si l'on a pour but une classification syntaxique des phrases: les phrases effectivement réalisées, issues de ce noyau abstrait, comportent des variantes de sens et des propriétés syntaxiques différentes qui sont attachées à ces variantes. Toutes ces données seraient mélangées sans qu'il soit possible de décrire les liens qui unissent les unes aux autres. Par exemple, dans le cas ci-dessus, le substantif attaché au verbe $(V-n=: a r m e)$, permet de construire une phrase associée à (1):

Les flics ont enlevé son arme au forcené

mais peut difficilement être relié à la construction (2):

?*Les arguments de Paul ont enlevé son arme à Marie

Lorsque le $V$ - $n$ est au pluriel, la situation est moins claire, dans la mesure où le $V-n$ subit aussi, dans ce cas, le glissement métaphorique (Marie a rendu les armes pourrait être un équivalent dans les deux sens).

D'autres propriétés sont attachées à l'un des emplois et pas à l'autre, par exemple la possibilité d'avoir pour (2) la phrase dérivée Paul est désarmant, qu'on ne peut rattacher à l'emploi (1): Les flics sont désarmants est clairement une phrase de type (2). Ces différences de comportement, très fréquentes, sont évidemment intéressantes à souligner, et le fait de séparer les entrées rend la description beaucoup plus claire et opératoire.

D'autres critères jouent en faveur du dédoublement: d'abord le fait que, dans le cas d'un sujet humain en (2), on a une phrase ambiguë :

Paul a désarmé Marie

qui peut avoir les deux sens: "propre " et "psychologique". Ce critère de l'ambiguïté, reproductible et donc opératoire, ne peut jouer que lorsque les distributions des actants, bien que différentes, ont une partie commune (ici $N O$ $=:$ Nhumain). Il en va de même pour les métaphores nominales souvent citées du type Paul est un lion: Paul peut être réellement le nom d'un fauve (c'est plus difficile, mais pas impossible, dans le cas de Marie est une perle). 
Un autre argument en faveur du dédoublement réside dans la constatation, pragmatique celle-ci, que l'emploi (2), de par son comportement syntaxique et distributionnel, appartient à la classe "naturelle " des verbes "psychologiques» de la table 4, comme amuser, étonner, dérouter, désarçonner, etc. Créer une entrée particulière pour le désarmer "psychologique " permet de compléter cette classe de verbes qui partagent plusieurs propriétés syntaxique. Notons que les deux derniers verbes cités ont aussi leur origine dans des métaphores claires : dérouter et désarçonner ont été dédoublés pour les mêmes raisons que désarmer.

Le cas de étonner, dans cette classe des verbes psychologiques, mérite une remarque particulière. Il est souvent fait appel à une approche diachronique pour justifier l'attribution de l'étiquette "sens propre": le sens propre de étonner, dans cette optique, serait "frapper par le tonnerre" et le sens psychologique de L'attitude de Paul étonne Marie serait métaphorique. Peut-on, en synchronie, parler de métaphore d'un emploi qui n'existe plus? La question se pose bien sûr pour le latin, mais, en disant cela, on ne fait que déplacer la question sur le terrain historique, qui n'est pas le nôtre ici.

La métaphore, dans tous ces cas, est à l'origine d'un emploi devenu autonome, "institutionnalisé », que nous considérons comme un verbe différent.

L'emploi de chiffonner que j'ai pris en 1.2., comme exemple de dédoublement justifié par des critères formels, relève du même type d'analyse. L'intuition de différence de sens est claire entre chiffonner une feuille de papier et chiffonner quelqu'un. Le sens psychologique, sans doute figuré (ou métaphorique) au départ, a donné naissance à un verbe nouveau, lexicalisé. Mais si certains cas sont clairs, on voit bien que beaucoup d'autres relèvent d'une intuition de sens qui ne constitue pas une base solide pour justifier un dédoublement d'entrée. Appuyer la définition de la métaphore sur la définition d'un sens propre suppose que l'on puisse définir ce dernier, ce qui, la littérature l'atteste, ne peut être fait de façon scientifiquement satisfaisante.

\subsection{La métaphore comme propriété secondaire}

Dans beaucoup de cas, l'intuition de différence de sens est moins nette que dans les exemples évoqués ci-dessus : la variation de distribution par rapport à l'emploi ressenti comme "normal » apparaît comme un jeu sémantique possible. Considérons le cas simple du verbe rudoyer. Son emploi "premier», ou "propre", ou "normal " (les querelles terminologiques à propos de notions aussi mal définies paraissent assez vaines) a été classé dans la table $32 \mathrm{H}$, définie par la structure NO V N1hum (complément d'objet purement humain):

Paul rudoie son fils

Nous considérons que la possibilité d'avoir:

Paul rudoie sa voiture, son ordinateur 
relève d'une création métaphorique, et non d'un emploi autonome justifiant la création d'un deuxième verbe rudoyer, qui serait caractérisé, lui, par un objet non-humain. D'autres verbes de la table $32 \mathrm{H}$, par exemple choyer, invectiver, martyriser ou même tutoyer autorisent le même glissement métaphorique. Nous avons donc introduit une propriété $N 1=N$-humain concret (objet non-humain concret) dans cette table définie par $N 1=N h u m$ obl (objet obligatoirement humain). Avec cette nuance, par rapport à d'autres exemples cités plus haut, que l'intuition de différence de sens concerne moins le verbe lui-même que le substantif objet direct: rudoyer sa voiture ou son ordinateur, c'est leur attribuer le trait [+humain], la restriction de sélection n'est donc que superficiellement violée. Ceci n'est possible qu'avec certaines catégories de substantifs "humanisables", dont font partie, dans nombre de cas, des objets mécaniques comme l'ordinateur ou la voiture. Notons d'ailleurs que si cette métaphore est productive, elle n'est pas systématiquement possible dans cette classe de verbes: molester sa voiture est douteux et l'estourbir encore plus difficile.

Les exemples suivants, tirés d'un corpus, relèvent du même problème: tous mettent en jeu une construction verbale qui, intuitivement, exige un sujet humain et sont classés comme tels. Le fait qu'on puisse mettre le substantif non humain virus comme sujet relève d'un glissement métaphorique aisément compréhensible; le virus, organisme vivant, peut dans certains contextes prendre les connotations habituellement attachées au trait « humain »:

Cevirus

a une stratégie de...

oppose une résistance...

choisit de...

déjoue les stratégies de défense...

survit...

est incapable de...

a un comportement atypique...

choisit l'homme comme hôte potentiel...

Ces cas seront traités dans les tables comme des métaphores ne justifiant pas un dédoublement d'emploi.

Le principe général qui régit le codage des métaphores possibles dans les tables du Lexique-Grammaire est donc le suivant : toute propriété distributionnelle en contradiction avec la définition d'une table de verbes indique la possibilité d'une création «déviante » métaphorique.

\subsection{Familles de métaphores}

Les tables syntaxiques correspondent souvent à des classes sémantiques «naturelles ", c'est-à-dire qu'elles forment une "famille » présentant une unité sémantique. À cette famille correspondent souvent des familles de métaphores productives. Citons-en quelques cas. 
Dans le grand groupe des constructions locatives, qui comporte plusieurs centaines de verbes (Guillet-Leclère 1992), toutes les constructions comportent un substantif représentant un lieu et un substantif dénotant un "corrélat " de ce lieu (l'objet situé par rapport à ce lieu). Plusieurs classes y sont distinguées, en fonction de la place syntaxique qu'occupent respectivement le lieu et son corrélat (sujet, objet direct ou complément prépositionnel). Plusieurs métaphores sont possibles, qui consistent en des variations de traits sémantiques sur tel ou tel couple d'actants. Ces variations peuvent être les mêmes dans les différentes constructions, en ce sens qu'elles concernent les mêmes emplacements thématiques que le substantif normal et se retrouvent donc dans les différentes tables lorsque cet emplacement change. Par exemple, dans la table $38 \mathrm{LD}$, le corrélat du lieu est en position d'objet direct et le lieu en position prépositionnelle, alors que c'est l'inverse dans les tables $37 \mathrm{M}$ :

Paul répand des graviers dans son allée (38LD)

Paul sème son jardin de fleurs (37M)

Dans la construction «normale» des verbes de ces classes, N1 et N2 sont des concrets. Mais nous avons constaté une série de métaphores productives qu'il nous a paru intéressant de noter car elles constituent des classes ${ }^{3}$ :

Ncorrélat $=N "$ "mot" et Nlieu $=N "$ "texte":

Paul a répandu des mots orduriers dans son texte (38LD)

Paul a semé son texte de citations latines (37M)

Ncorrélat $=$ N"idée" et Nlieu $=N$ "esprit":

Paul a enfoui ce souvenir au fond de sa mémoire (38LD)

Paul encombre son esprit de notions sans intérêt (37M)

Dans le cas où l'un des actants est un humain et l'autre un Nabstrait, par contre, il n'y a pas interversion des rôles; l'humain est le corrélat du lieu en 38LD et le lieu dans 37M :

Cette situation a englué Paul dans ses problèmes (38LD)

On a imbibé Paul de principes réactionnaires (37M)

Les variations sur les traits attachés aux actants ne concernent pas seulement les grandes catégories comme humain/non humain. Il peut s'agir de traits plus précis, qui n'ont de sens que pour une classe particulière d'actants et dont le codage permet $d$ 'isoler des familles de constructions qu'on pourrait appeler métaphoriques.

Considérons par exemple la table $38 \mathrm{~L} 1$ : la construction définitionnelle en est $N O V N 1$, avec $N O=$ Ncorrélat et $N 1=$ Nlieu. Dans le sens "normal " des verbes de cette classe, le corrélat est un objet en mouvement:

Le rayon balaye l'écran; la voiture percute le platane; l'eau envahit le jardin

Le camion longe la rivière, gravit la colline, atteint le sommet

3. Le nom entre guillemets est un substantif classifieur représentant une classe de substantifs. 
Les verbes de la deuxième série acceptent comme sujet un substantif dénotant le "chemin » sur lequel peut se déplacer un objet en mouvement:

La route longe la rivière, gravit la colline, atteint le sommet

L'interprétation «statique » de ces constructions constitue une déviance qui a été codée en colonne. La même situation s'observe avec les constructions prépositionnelles:

Paul sort de Paris, aboutit à Lyon

La route sort de Paris, aboutit à Lyon (interprétation locative dynamique)

(interprétation locative statique)

Il n'est pas clair pourtant qu'il s'agisse ici de métaphore. On pourrait tout aussi bien parler de déviance métonymique (le "chemin " pour le véhicule qui roule dessus) donnant naissance à un emploi autonome statique du verbe.

\section{Un cas particulier de distribution : la métonymie}

Quand on parle de la distribution des actants et des traits sémantiques qui leur sont attachés, il convient de faire la distinction entre les mots tels qu'ils apparaissent en surface dans la phrase et les constructions plus profondes dont ils sont éventuellement dérivés, ou qui sous-tendent le sens qu'ils ont dans tel ou tel environnement. On trouve là encore un argument en faveur de la thèse, défendue dans le Lexique-Grammaire, que l'unité de base pour l'interprétation sémantique est la phrase. Dans l'exemple:

Max a amusé tout le wagon avec ses calembours (Guillet 1986)

il ne s'agit pas d'une métaphore consistant en un glissement de l'objet direct, de la catégorie Nhumain à N-humain. Le wagon est tout simplement, dans ce cas, un Ncollectif humain qu'on peut décomposer en : les gens du wagon. On retrouve ici le même type de métonymie que dans le cas bien connu:

Washington a décidé d'intervenir

dérivé de le gouvernement de Washington, ou de le gouvernement des États-Unis siégeant à Washington, qui respectent la contrainte "sujet humain" attachée au verbe décider.

De la même façon, la contrainte "complément d'objet obligatoirement au pluriel », qui définit la classe des verbes de la table 32PL, comme accumuler ou feuilleter, n'est pas violée dans des exemples comme:

Paul a accumulé un trésor de guerre

Paul a feuilleté le magazine

Bien que formellement singulier, le complément est ici sémantiquement pluriel. On ne peut avoir:

*Paul a accumulé un louis d'or

* Paul a feuilleté la page 46 
Notons que ces déviances superficielles, qui ne relèvent pas d'une production métaphorique, posent des problèmes pour l'analyse automatique, qui ne peut prendre en compte que le critère formel du pluriel.

Il existe bien d'autres cas où la détermination des traits attachés à un actant, dans une phrase donnée, dépend de la prise en compte d'une phrase sous-jacente plus complexe. Schématiquement, quand, à une place syntaxique, on peut avoir de façon équivalente soit un groupe nominal [ $\mathrm{Na}$ de $N b$ l, soit un $N$ de même sens, lequel faut-il prendre en compte pour définir la distribution «normale» du verbe? Il n'y a malheureusement pas de réponse satisfaisante à cette question, elle dépend du verbe et du type de noms mis en jeu dans la phrase:

"The apparent shift in the meaning of words are due either to the different operators or arguments with which they are occurring, or to the retained meaning of a zeroed operator or argument; this is also the case for metaphoric occurrences " (Harris 1988).

Considérons les verbes amuser et émouvoir, par exemple. Ils acceptent:

(1) Paul a amusé [les gens du wagon]

(2) Cette histoire a ému [le coeur de Jean]

Décider de classer ces deux verbes dans la table 4 (verbes psychologiques à objet direct purement humain) passe par une analyse des groupes nominaux qui déterminera que dans (1), c'est $N a$ qui est la " tête sémantique » du $G N$, alors que c'est $N b$ dans (2). Cette analyse n'est pas toujours facile.

Ce type de problème se pose en permanence et met en jeu de nombreuses données linguistiques (notions de substantif approprié effaçable, de restructuration du groupe nominal, etc.). Chaque fois qu'une relation syntaxique est établie clairement entre deux phrases de même sens, on considérera que les restrictions de sélection du verbe ne sont pas violées, et que donc il n'y a pas métaphore.

\section{Conclusion}

Si les auteurs tournent sans fin autour de sa définition, c'est que la métaphore échappe à toute tentative de délimitation précise, d'abord à cause des multiples formes qu'elle peut prendre, ensuite parce qu'elle constitue un phénomène de productivité continuelle du langage, inséparable de la dimension historique. Il y a métaphore dès que l'on sort de la phrase basique donnant le sens "propre " d'un mot, sens qu'on ne connaît pas toujours. Dire que le sens propre de tel mot est $x$, qui vient du latin $y$, c'est supposer que le latin représente la «normalité » et est un état stable de la langue. Mais rien ne dit qu'il ne

4. Je remercie Takuya Nakamura de m'avoir fourni plusieurs documents utiles sur le sujet. 
s'agissait pas déjà d'une métaphore en latin. Si plonger est, comme on le dit, dérivé de plombum (plomb), l'emploi propre est quelque chose comme couler comme du plomb et tous les emplois modernes sont métaphoriques...

Dans beaucoup de cas, pourtant, le sentiment de métaphore existe bel et bien. Mais il est intuitif et peut dépendre de la culture du sujet parlant.

Si l'on ne peut définir de façon satisfaisante la frontière entre métaphore et emploi autonome d'un mot (et on ne le peut pas), l'important pour nous est que toutes les possibilités de réalisation de ce mot dans des phrases soient indiquées dans le Lexique-Grammaire, que ce soit sous forme d'entrées à part entière ou de simples propriétés dans une classe de verbes.

\section{Bibliographie}

BOONS, Jean-Paul, GuILLET, Alain, LECLÈRE, Christian, 1976a : La structure des phrases simples en français. Constructions intransitives. Genève: Droz.

BOONS, Jean-Paul, GuILLET, Alain, LECLĖRE, Christian, 1976b : La structure des phrases simples en français. Classes de constructions transitives. Rapport de Recherches 6, Paris: LADL.

BoONs, Jean-Paul, 1971 : Métaphore et baisse de la redondance. Langue Française 11, Paris: Larousse.

FONTANIER, Pierre, 1968 : Les figures du discours. Paris: Flammarion.

GuILLET, Alain, 1986: Représentation des distributions dans un lexique-grammaire. Langue Française 69, Paris: Larousse.

Guillet, Alain, 1990 : Fondements formels des classes sémantiques dans un lexiquegrammaire. Langages 98, Paris: Larousse.

GUILLET, Alain, LECLÈRE, Christian, 1992: La structure des phrases simples en français. Les constructions transitives locatives. Genève: Droz.

Gross, Gaston, 1994 : Classes d'objet et description des verbes. Langages 115, Paris: Larousse.

GROSS, Maurice, 1975: Méthodes én syntaxe. Paris : Hermann.

HARRIS, Z., 1988 : Language and Information, New York: Columbia University Press. 